\title{
Hemodynamic Characteristics Regarding Recanalization of Completely Coiled Aneurysms: Computational Fluid Dynamic Analysis Using Virtual Models Comparison
}

\author{
Wonhyoung Park, MD',2, Yunsun Song, MD', Kye Jin Park, MD', Hae-Won Koo, MD', \\ Kuhyun Yang, MD', Dae Chul Suh, MD, PhD'
}

Purpose: Hemodynamic factors are considered to play an important role in initiation and progression of the recurrence after endosaccular coiling of the intracranial aneurysms. We made paired virtual models of completely coiled aneurysms which were subsequently recanalized and compared to identify hemodynamic characteristics related to the recurred aneurysmal sac.

Materials and Methods: We created paired virtual models of computational fluid dynamics (CFD) in five aneurysms which were initially regarded as having achieved complete occlusion and then recurred during follow-up. Paired virtual models consisted of the CFD model of 3D rotational angiography obtained in the recurred aneurysm and the control model of the initial, parent artery after artificial removal of the coiled and recanalized aneurysm. Using the CFD analysis of the virtual model, we analyzed the hemodynamic characteristics on the neck of each aneurysm before and after its recurrence.

Results: High wall shear stress (WSS) was identified at the cross-sectionally identified aneurysm neck at which recurrence developed in all cases. A small vortex formation with relatively low velocity in front of the neck was also identified in four cases. The aneurysm recurrence locations corresponded to the location of high WSS and/or small vortex formation.

Conclusion: Recanalized aneurysms revealed increased WSS and small vortex formation at the cross-sectional neck of the aneurysm. This observation may partially explain the hemodynamic causes of future recanalization after coil embolization.

Key Words: Embolization; Hemodynamics; Intracranial aneurysm; Recurrence; Wall shear stress

'Department of Radiology, University of Ulsan College of Medicine, Asan Medical Center, Seoul, Korea

${ }^{2}$ Department of Neurosurgery, University of Ulsan College of Medicine, Asan Medical Center, Seoul, Korea

Received February 24, 2016; accepted after revision February 25, 2016.

Correspondence to: Dae Chul Suh, MD, PhD, Department of Radiology, University of Ulsan College of Medicine, Asan Medical Center, 88 Olympic-ro 43-gil, Songpa-gu, Seoul 05505, Korea.

Tel. 82.2.3010.4366 Fax.82.2.476.0090 E-mail: dcsuh@amc.seoul.kr

This is an Open Access article distributed under the terms of the Creative Commons Attribution Non-Commercial License (http://creativecommons.org/licenses/by-nc/3.0) which permits unrestricted non-commercial use, distribution, and reproduction in any medium, provided the original work is properly cited. 
As endovascular treatment techniques and materials advance in their development, an increasing number of intracranial aneurysms have been treated using these methods. Endovascular treatment of intracranial aneurysms is regarded as a minimally invasive and reliable treatment modality with low patient morbidity and mortality. However, aneurysm recanalization after coil embolization remains a major problem. Several studies have reported relatively high recanalization rates during the follow-up period in $8-33.6 \%$ of the aneurysm patients treated using detachable coils [1-5]. In cases of completely occluded aneurysms following the initial coil embolization, the incidence of recanalization during the follow-up period has been reported as $12.7-14 \%[1,5]$. Several factors, such as congenital vascular defect, hypertension, smoking, intramural thrombus, ruptured aneurysm, large-sized aneurysm, aneurysm under-packing, and residual neck after treatment may be considered as contributing to recanalization following coil embolization [1,5-11]. Several studies have also reported that the blood hemodynamics may have a profound impact on the development of recanalization after coil embolization of intracranial aneurysms using computational fluid dynamics (CFD) [10, 12-14].

Recent studies regarding the blood hemodynamics of coil-packed aneurysms were based on patient-specific, 3-dimensional (3D), digital subtraction angiography (DSA) data or 3D magnetic resonance angiography (MRA) $[10,12,14,15]$. As a result of CFD analysis based on patient-specific 3D DSA, increased wallshear stress (WSS) at the necks of completely occluded aneurysms after coil embolization may be an important hemodynamic factor that influences recanalization during the follow-up period [12]. However, a coilrelated artifact sometimes makes it difficult to evaluate whether or not there is a tiny residual neck after embolization, when seen on 3D DSA or 3D MRA. Therefore, we made new virtual models of completely occluded aneurysms which were based on 3D DSA of real patients with recurred aneurysms after coil embolization in order to overcome these biases. We also conducted CFD analysis using these new virtual models of completely occluded aneurysms in order to identify hemodynamic risk factors that may affect the recanalization.

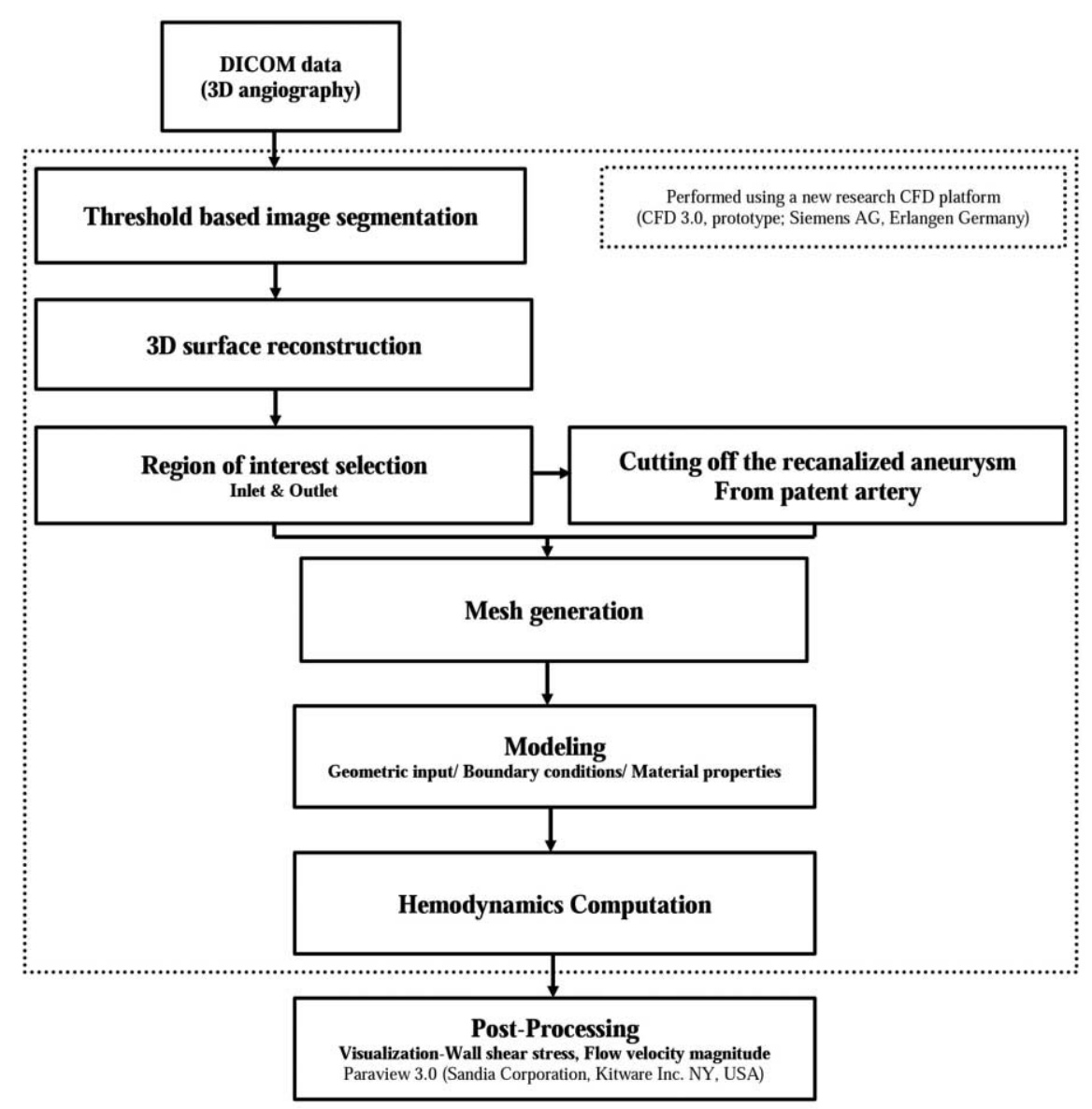

Fig. 1. Flow sheet of the computational fluid dynamics analysis using new virtual models of completely occluded aneurysms based on patient specific imaging data. 


\section{MATERIALS AND METHODS}

\section{Patients and imaging data acquisition}

This study was approved by our institutional review board. The five, intracranial, recanalized aneurysms were retrospectively identified in five patients from our neurointerventional database who underwent coil embolization without stenting and had follow-up 3D DSA data. The aneurysms were regarded as having achieved complete occlusion according to the modified Raymond classification [3]. The mean size (SD) of the aneurysms before coil embolization was $7.4 \mathrm{~mm}$ (2.4). The aspect ratios of the aneurysms were as follows: 1.87 in case $1 ; 1.43$ in case $2 ; 2.74$ in case $3 ; 1.14$ in case 4 ; and 2.13 in case 5 . The recanalized aneurysms were located at the origin of the posterior communicating artery in cases 3 and 4, at the basilar tip in case 1, at the anterior communicating artery in case 2 and at the paraclinoid internal carotid artery (ICA) in case 5 . There were two ruptured aneurysms (cases 1 and 3).
All of these aneurysms were treated using coil embolization without stenting. All of the aneurysms showed recanalization during the follow-up period, and the mean follow-up period ( \pm SD) was 18.0 months $( \pm 12.9)$. MRA is generally used for follow-up after coil embolization and if the MRA shows recanalization of the treated aneurysm, 3D DSA is performed in order to confirm the recanalization. 3D DSA was obtained on AXIOM Artis Zee (Siemens AG, Erlangen, Germany), and the 3D data were available as DICOM data after reconstruction on an X-Workplace (Siemens AG, Erlangen, Germany).

\section{Paired virtual models and CFD analysis}

As we described in our previous study, we used a new CFD platform (CFD 3.0, prototype- not for diagnostic use; Siemens AG, Erlangen, Germany) for CFD analysis [16]. The flow sheet of this analysis is summarized in Figure 1. The DICOM data were transferred to this platform. Using this platform, all of the integral steps for producing a 3D model of the
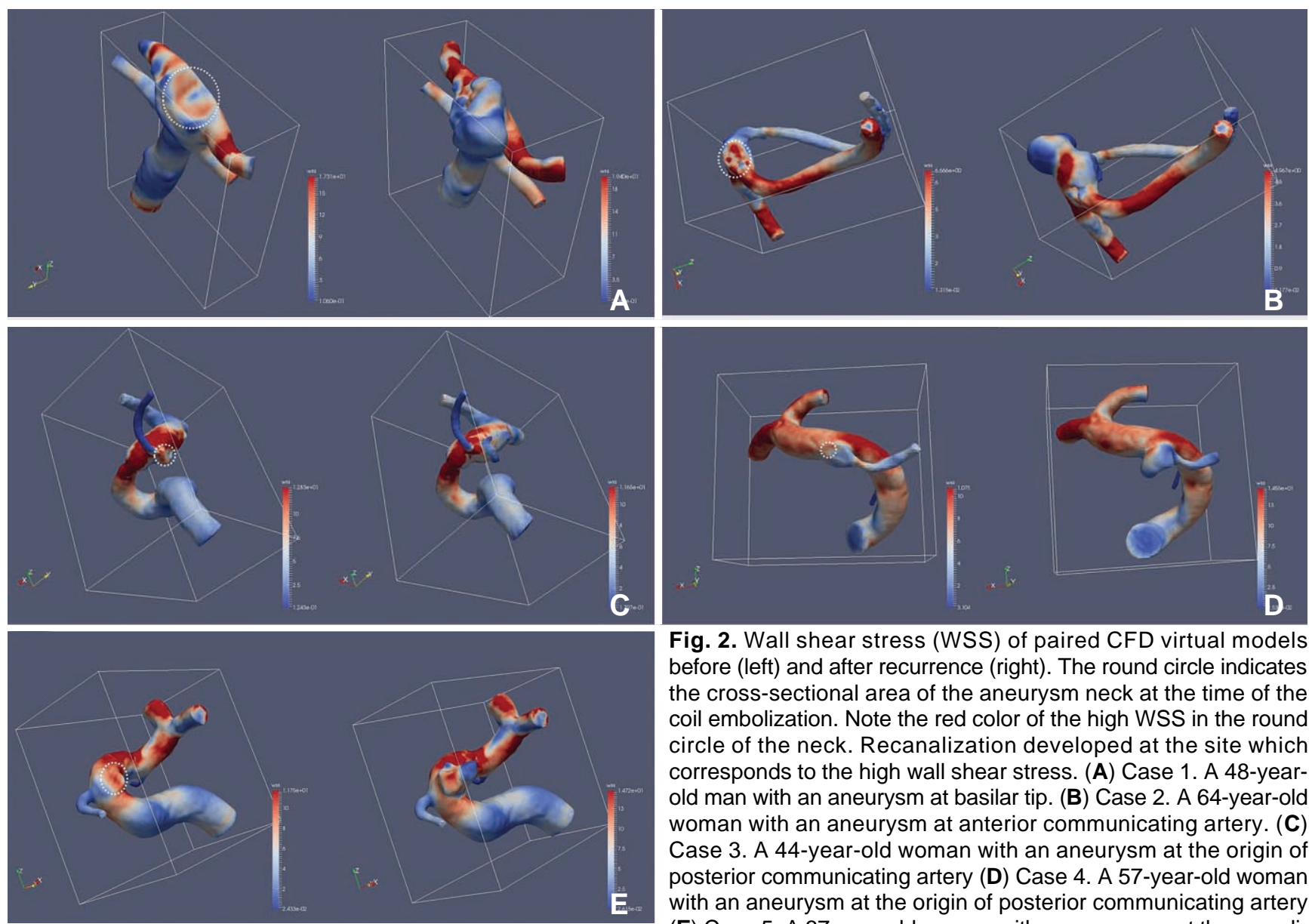

Fig. 2. Wall shear stress (WSS) of paired CFD virtual models before (left) and after recurrence (right). The round circle indicates the cross-sectional area of the aneurysm neck at the time of the coil embolization. Note the red color of the high WSS in the round circle of the neck. Recanalization developed at the site which corresponds to the high wall shear stress. (A) Case 1. A 48-yearold man with an aneurysm at basilar tip. (B) Case 2. A 64-year-old woman with an aneurysm at anterior communicating artery. (C) Case 3. A 44-year-old woman with an aneurysm at the origin of posterior communicating artery (D) Case 4. A 57-year-old woman with an aneurysm at the origin of posterior communicating artery (E) Case 5. A 37-year-old woman with an aneurysm at the paraclinoid internal carotid artery. 
patient-specific vascular structure are available, including the initial DICOM processing, segmentation, and surface-mesh generation. During the step of surfacemesh generation, we could create a paired model of one patient that consisted of a completely occluded aneurysm model and recanalized model based on patient-specific 3D DSA data using this research CFD platform. The virtual model of the completely occluded aneurysm was generated by artificially cutting off the recanalized aneurysm from the parent artery using this CFD platform. After this process, the virtual arterial wall under the identical conditions as the normal arterial wall was generated for the cross-section of the artificial cutting. In the next step, the inlet and outlets of the target arterial structure were designated in order to define the boundaries. The results were then saved in specific files in the STL format in order to progress to the next steps using this research CFD platform, although they could also be exported elsewhere. Using this protocol, we could create two 3D models of vascular structures, i.e. a completely occluded aneurysm model and a recanalized aneurysm model, in the same patient, based on the DICOM data of 3D DSA which were obtained so as to confirm the recanalization of the treated aneurysm.

This new research CFD platform also includes the solver which is based on the "Lattice Boltzmann Method", a promising numerical scheme used for simulating complex fluid dynamics in the most varied applications, including models of the cerebral artery $[17,18]$. The essential step of surface-mesh generation for the simulation domain was automatically performed and also integrated into the solver. A Newtonian flow condition was used to perform calculation for CFD analysis. Calculations were performed using the following material constants: the density and dynamic viscosity of blood as $0.001 \mathrm{~g} / \mathrm{mm}^{3}$ and 0.004 Poiseuille ( $\mathrm{Pa}$ $\mathrm{S})$; the number of cardiac cycles (default, 2); and the output frequency (default, $0.05 \mathrm{~s}$ ). The velocity profile of the inlet was defined as pulsatile, and which corresponded to a pulse length of $0.87 \mathrm{~s}$ and minimum and maximum flow velocities of $12.7 \mathrm{~cm} / \mathrm{s}$ and 30.0 $\mathrm{cm} / \mathrm{sec}$, respectively. We used the average Reynolds number which was within the range of normal blood flow in human arteries, as had been used in previous studies $[10,12,14,19]$. Zero pressure boundary conditions were prescribed at all outlets. In addition, the blood-vessel wall was assumed to be rigid with a no-slip boundary condition.

\section{Visualization and comparison of the hemodynamic factors}

The flow velocity magnitude and the WSS at the systolic peak around the neck of the aneurysm were calculated, and the results were saved in specific files as VTK format for visualization. Visualization of the results was performed using Paraview 3.0 (Sandia Corporation, Kitware Inc. NY, USA) which could allow comparison of the two data sets at the same view and rotation angles by pixel-by-pixel bases. Flowvelocity magnitudes around the neck of the aneurysms at the systolic peak were visualized with instantaneous streamlines as flow patterns in the 3D space using glyph rendering, and the WSSs around the neck of the aneurysm were also visualized with a color map in the $3 \mathrm{D}$ space using surface rendering. These qualitative results of hemodynamic factors were obtained from CFD analysis of the completely occluded aneurysm model and recanalized aneurysm model in the same patient. Finally, we evaluated the correlation between the hemodynamic factors and recanalization of the aneurysm following coil embolization.

\section{RESULTS}

The area with increased WSS at the systolic peak of
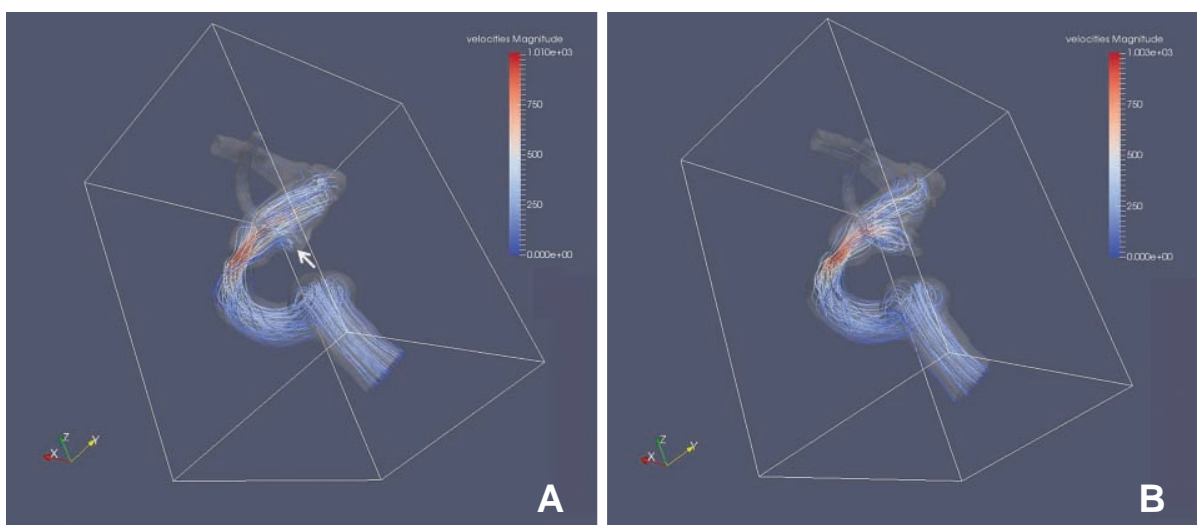

Fig. 3. Blood flow of paired CFD virtual models before and after recurrence in case 3. (A) White arrow indicates a small vortex formation near the neck of completely occluded aneurysm at the origin of posterior communicating artery. (B) The blood flow entered the sac of recanalized aneurysms through the impingement region and it formed a large and complex vortex in the recanalized aneurysmal sac. 
the cross-sectionally oriented neck of completely occluded aneurysms was observed in five cases (Fig. 2 ). The flow-velocity magnitude at the systolic peak around the neck of the completely occluded aneurysm was not increased in all five cases. However, a small vortex formation with relatively low velocity near the neck of a completely occluded aneurysm was identified in four cases, except for case 5 (Fig. 3). The increased WSS and the small vortex formation developed at almost the same locations in four cases, except for case 5. In addition, the recanalization points corresponded almost exactly to the location of the increased WSS and/or the small vortex in all five cases. Finally, the blood flow entered the sac of recanalized aneurysms through the impingement region and it formed a large and complex vortex in the recanalized aneurysmal sac (Fig. 3).

\section{DISCUSSION}

Several studies have reported recanalization of a completely coiled aneurysm as modified Raymond scale 1, despite the best efforts of neurointerventionists $[5,20]$. Hemodynamic features around the perianeurysmal regions may have an important role in bringing about the recanalization of a coiled aneurysm, such as other risk factors including the aneurysm characteristics, patient characteristics, and technical problems. Therefore, we performed CFD analysis of the local hemodynamic features both before and after recanalization using novel virtual models. Our study shows that in completely coiled aneurysms that resulted in recanalization, there were increased WSS and a small vortex formation with a relatively low velocity at the cross-sectionally oriented neck of coiled aneurysms.

The previous studies regarding the CFD analysis of coil-embolized aneurysms were based on computergenerated aneurysm models, not on patient-specific aneurysm models, and which may result in a potential bias in the following computational simulation analyses [21-23]. For example, some studies made the geometry of parent arteries and branches as straight without flexures and diameter variation and made the aneurysms with a smooth surface and almost globular shape [21, 23]. In order to overcome these limitations, recent CFD analysis studies of the hemodynamics around coil-packed aneurysms were based on patientspecific data derived from 3D DSA or 3D MRA [10, $12,14,15]$. Since using the patient-specific data, the CFD analysis for hemodynamic factors has been made possible in various structures of parent arteries and aneurysms. However, a previous study that performed CFD analysis for identifying hemodynamic features on recanalization used the 3D DSA data of completely coiled aneurysms as the modified Raymond scale [1, 12]. However, several studies have reported that detachable coils in the treated aneurysm may cause an artifact which sometimes makes it difficult to evaluate whether the tiny residual neck is seen on 3D DSA or 3D MRA [24-26]. Therefore, we decided to create new virtual models of completely occluded aneurysms based on patient-specific 3D DSA data in order to remove the possible presence of a residual neck following coil embolization.

Several recent studies have focused on the hemodynamic patterns of embolized aneurysms with a residual neck using CFD analysis based on patient-specific imaging data. These studies reported that high WSS and/or high blood flow velocity were identified near the residual neck of partially embolized aneurysms susceptible to future recanalization $[10,14,15]$. However, there have only been a few studies which attempted to identify the hemodynamic patterns which lead to the recurrence of totally embolized aneurysms, using CFD analysis based on patient-specific imaging data. Li et al. evaluated the hemodynamic features at the aneurysm neck using CFD analysis with patientspecific 3D DSA, while attempting to identify the hemodynamic risk factors leading to the recurrence of completely embolized aneurysms without residual lesions [12]. They discovered that increased WSS at the completely occluded aneurysm neck after embolization may be an important risk factor that contributes to recanalization of an aneurysm. In our study, there were points at which there was increased WSS at the systolic peak near the neck of completely occluded aneurysms in all five cases. In canine models, disruption of internal elastic lamina, loss of medial smooth-muscle cells, reduced proliferation of smooth-muscle cells, and loss of fibronectin were observed at the regions with higher WSS than at the normal physiological levels [27]. Other studies also claimed that WSS higher than the normal physiologic level may disrupt the normal function of end othelial cells [28, 29]. Therefore, increased WSS may impede neointimal formation which is induced by coil placement. Byun et al. also reported that high WSS after coil embolization may disrupt thrombus formation in embolized aneurysms [30]. Finally, our study supported that increased WSS near the neck of a completely embolized aneurysm can cause future recanalization probably via disruption of 


\section{Aneurysm Recanalization and Hemodynamic Features}

the neointima formation of the neck as well as impediment of thrombus formation into the aneurysmal sac.

In our study, a small vortex formation with relatively low velocity near the neck of a completely occluded aneurysm was identified in four cases. This phenomenon indicates that there are certain amount of energy loss leading to a small vortex formation and subsequent stagnation of blood flow near the neck of a completely occluded aneurysm. Several studies have reported that there was a significant relationship between the focal endothelial injury and the stagnation of blood flow [31, 32]. In addition, Nakatani et al reported that the stagnation of blood flow may cause anoxic injury to endothelial cells and accumulation of platelets or leukocytes that damage intimal tissue [31]. Therefore, stagnation of blood flow may interfere with neointima formation of the neck of a completely embolized aneurysm.

Our study has some limitations. First, we obtained the control model for comparison by removing the coil mass and recurred aneurysm and which may differ from the initial vessel status after coil embolization. However, there is also an advantage to obtaining better CFD results by avoiding metal artifacts which usually makes CFD analysis difficult at the neck. Second, our sample size was relatively small as only five patients were included in this study. Further studies should be performed in a large study population using our methodology. Third, quantitative analysis was not applied in our study. However, this newly developed CFD platform could provide rapid processing and comparison of the results even though it was qualitative. Fourth, several assumptions were made regarding the CFD analysis, including the Newtonian flow condition, zero pressure boundary condition at all of the outlets, and the rigid with no-slip boundary condition of the arterial wall. However, discrepancies resulting from such an assumption can be compensated for paired CFD model comparison with the control.

\section{CONCLUSION}

Our study shows that increased WSS and small vortex formation were identified in the cross-sectional area of the neck in completely occluded aneurysms using paired virtual CFD modeling methods. This observation may increase the evidence regarding the hemodynamic characteristics which can explain the recanalization mechanism in completely occluded aneurysms by coiling. CFD analysis using patientspecific 3D DSA may be helpful in order to identify coil-embolized aneurysms which have a risk of recanal- ization during the follow-up period.

\section{References}

1. Cognard C, Weill A, Spelle L, Piotin M, Castaings L, Rey A, et al. Long-term angiographic follow-up of 169 intracranial berry aneurysms occluded with detachable coils. Radiology 1999; 212:348-356

2. Ng P, Khangure MS, Phatouros CC, Bynevelt M, ApSimon H, McAuliffe W. Endovascular treatment of intracranial aneurysms with Guglielmi detachable coils: analysis of midterm angiographic and clinical outcomes. Stroke 2002;33:210-217

3. Raymond J, Guilbert F, Weill A, Georganos SA, Juravsky L, Lambert A, et al. Long-term angiographic recurrences after selective endovascular treatment of aneurysms with detachable coils. Stroke 2003;34:1398-1403

4. Park SH, Lee CY, Yim MB. The merits of endovascular coil surgery for patients with unruptured intracranial aneurysms. $J$ Korean Neurosurg Soc 2008;43:270-274

5. Grunwald IQ, Papanagiotou P, Struffert T, Politi M, Krick C, Gul $\mathrm{G}$, et al. Recanalization after endovascular treatment of intracerebral aneurysms. Neuroradiology 2007;49:41-47

6. Boet R, Wong GK, Poon WS, Lam JM, Yu SC. Aneurysm recurrence after treatment of paraclinoid/ophthalmic segment aneurysms--a treatment-modality assessment. Acta Neurochir (Wien) 2005;147:611-616; discussion 616

7. Ortiz R, Stefanski M, Rosenwasser R, Veznedaroglu E. Cigarette smoking as a risk factor for recurrence of aneurysms treated by endosaccular occlusion. J Neurosurg 2008;108:672-675

8. Plowman RS, Clarke A, Clarke M, Byrne JV. Sixteen-year singlesurgeon experience with coil embolization for ruptured intracranial aneurysms: recurrence rates and incidence of late rebleeding. Clinical article. J Neurosurg 2011;114:863-874

9. Byrne JV, Sohn MJ, Molyneux AJ, Chir B. Five-year experience in using coil embolization for ruptured intracranial aneurysms: outcomes and incidence of late rebleeding. J Neurosurg 1999; 90:656-663

10. Luo B, Yang X, Wang S, Li H, Chen J, Yu H, et al. High shear stress and flow velocity in partially occluded aneurysms prone to recanalization. Stroke 2011;42:745-753

11. Lecler A, Raymond J, Rodriguez-Regent C, Al Shareef F, Trystram D, Godon-Hardy S, et al. Intracranial Aneurysms: Recurrences More than 10 Years after Endovascular Treatment-A Prospective Cohort Study, Systematic Review, and MetaAnalysis. Radiology 2015;277:173-180

12. Li C, Wang S, Chen J, Yu H, Zhang Y, Jiang F, et al. Influence of hemodynamics on recanalization of totally occluded intracranial aneurysms: a patient-specific computational fluid dynamic simulation study. J Neurosurg 2012;117:276-283

13. Ortega J, Hartman J, Rodriguez J, Maitland D. Post-treatment hemodynamics of a basilar aneurysm and bifurcation. Ann Biomed Eng 2008;36:1531-1546

14. Irie K, Anzai H, Kojima M, Honjo N, Ohta M, Hirose Y, et al. Computational fluid dynamic analysis following recurrence of cerebral aneurysm after coil embolization. Asian J Neurosurg 2012; 7:109

15. Liu J, Jing L, Wang C, Paliwal N, Wang S, Zhang Y, et al. Effect of hemodynamics on outcome of subtotally occluded paraclinoid 


\section{Wonhyoung Park, et al.}

aneurysms after stent-assisted coil embolization. $J$ Neurointerv Surg 2015; J Neurointerv Surg. 2015 Nov 26. doi: 10.1136/ neurintsurg-2015-012050. [Epub ahead of print]

16. Song Y, Choe J, Liu H, Park KJ, Yu H, Lim OK, et al. Virtual stenting of intracranial aneurysms: application of hemodynamic modification analysis. Acta Radiol 2015; Acta Radiol. 2015 Oct 25. [Epub ahead of print]

17. He X, Duckwiler G, Valentino DJ. Lattice Boltzmann simulation of cerebral artery hemodynamics. Computers \& Fluids 2009; 38:789-796

18. Golbert DR, Blanco PJ, Clausse A, Feijoo RA. Tuning a latticeBoltzmann model for applications in computational hemodynamics. Med Eng Phys 2012;34:339-349

19. Mitsos AP, Kakalis NM, Ventikos YP, Byrne JV. Haemodynamic simulation of aneurysm coiling in an anatomically accurate computational fluid dynamics model: technical note. Neuroradiology 2008;50:341-347

20. Gallas S, Januel AC, Pasco A, Drouineau J, Gabrillargues J, Gaston A, et al. Long-term follow-up of 1036 cerebral aneurysms treated by bare coils: a multicentric cohort treated between 1998 and 2003. AJNR Am J Neuroradiol 2009;30:1986-1992

21. Cha KS, Balaras E, Lieber BB, Sadasivan C, Wakhloo AK. Modeling the interaction of coils with the local blood flow after coil embolization of intracranial aneurysms. J Biomech Eng 2007; 129:873-879

22. Cebral JR, Lohner R. Efficient simulation of blood flow past complex endovascular devices using an adaptive embedding technique. IEEE Trans Med Imaging 2005;24:468-476

23. Groden C, Laudan J, Gatchell S, Zeumer H. Three-dimensional pulsatile flow simulation before and after endovascular coil embolization of a terminal cerebral aneurysm. J Cereb Blood Flow Metab 2001; 21:1464-1471
24. Gauvrit JY, Leclerc X, Caron S, Taschner CA, Lejeune JP, Pruvo JP. Intracranial aneurysms treated with Guglielmi detachable coils imaging follow-up with contrast-enhanced MR angiography. Stroke 2006;37:1033-1037

25. Schaafsma JD, Velthuis BK, Majoie CBLM, van den Berg R, Brouwer PA, Barkhof F, et al. Intracranial aneurysms treated with coil placement: test characteristics of follow-up MR angiography-multicenter study. Radiology 2010;256:209-218

26. Missler U, Hundt C, Wiesmann M, Mayer T, Brückmann H. Three-dimensional reconstructed rotational digital subtraction angiography in planning treatment of intracranial aneurysms. Eur Radiol 2000;10:564-568

27. Meng H, Wang Z, Hoi Y, Gao L, Metaxa E, Swartz DD, et al. Complex hemodynamics at the apex of an arterial bifurcation induces vascular remodeling resembling cerebral aneurysm initiation. Stroke 2007;38:1924-1931

28. Chatziprodromou I, Tricoli A, Poulikakos D, Ventikos Y. Haemodynamics and wall remodelling of a growing cerebral aneurysm: a computational model. J Biomech 2007;40:412-426

29. Wang J, Tan HQ, Zhu YQ, Li MH, Li ZZ, Yan L, et al. Complex hemodynamic insult in combination with wall degeneration at the apex of an arterial bifurcation contributes to generation of nascent aneurysms in a canine model. AJNR Am J Neuroradiol 2014; 35:1805-1812

30. Byun HS, Rhee K. CFD modeling of blood flow following coil embolization of aneurysms. Med Eng Phys 2004;26:755-761

31. Nakatani H, Hashimoto N, Kang Y, Yamazoe N, Kikuchi H, Yamaguchi S, et al. Cerebral blood flow patterns at major vessel bifurcations and aneurysms in rats. J Neurosurg 1991;74:258-262

32. Mantha A, Karmonik C, Benndorf G, Strother C, Metcalfe R. Hemodynamics in a cerebral artery before and after the formation of an aneurysm. AJNR Am J Neuroradiol 2006; 27:1113-1118 